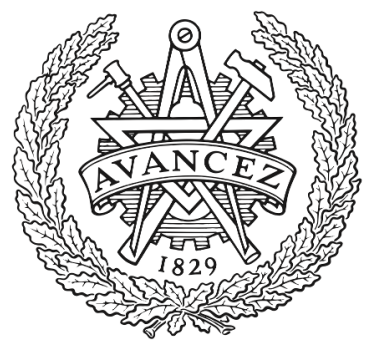

CHALMERS

UNIVERSITY OF TECHNOLOGY

\title{
Capacity scaling of flexible optical networks with nonlinear impairment [Invited]
}

Downloaded from: https://research.chalmers.se, 2023-04-26 11:25 UTC

Citation for the original published paper (version of record):

Yan, L., Agrell, E. (2017). Capacity scaling of flexible optical networks with nonlinear impairment [Invited]. International Conference on Transparent Optical Networks.

http://dx.doi.org/10.1109/ICTON.2017.8025167

N.B. When citing this work, cite the original published paper. 


\title{
Capacity Scaling of Flexible Optical Networks With Nonlinear Impairment
}

\author{
Li Yan and Erik Agrell
}

\begin{abstract}
Efficient resource utilization with a satisfying quality of service is the key to high capacity in flexible optical networks. In our work, this is achieved by supporting the resource allocation with the Gaussian noise model, which estimates the nonlinear physical-layer impairments. The study includes variable launch power, modulation formats, error-correcting code rates, and various new network features to provision traffic requests efficiently. The advantageous performance of the flexible optical networks is demonstrated by simulation results.
\end{abstract}

Keywords-Flexible optical networks, Gaussian noise model, resource allocation

\section{INTRODUCTION}

The rapid rise in the use of the Internet has led to increasing data volumes and diversified traffic requests, which put severe pressure on backbone optical networks. Flexible-grid optical networks have been developed to relax the rigid spectrum grid requirement of wavelength-division multiplexing (WDM) networks and offer much higher efficiency by adaptively assigning spectrum to traffic demands [1]. New transmission techniques and network features are also introduced into flexible-grid networks to further boost the capacity. Consequently, the states of flexible-grid networks become extremely complicated and physical layer impairments (PLIs) will be the dominant limiting factor for satisfactory qualities of transmission (QoTs). Hence, studying efficient and intelligent resource allocation algorithms that overcome these challenges is meaningful to avoid the so-called "capacity crunch" [2], [3].

To achieve acceptable QoTs in highly heterogeneous network environments, we need to consider the significant nonlinear interference (NLI) among demands coexisting on different wavelengths in the same fiber link [4]. Recently, the Gaussian noise (GN) model [5], [6] has been proposed to approximate the PLIs analytically with reasonable accuracy and low computational complexity. Based on this model, the demand signalto-noise ratio (SNR) can be estimated according to the overall network state. Significant efficiency improvements have been achieved in the optical networks by precise NLI evaluation and optimized resource allocation [7], [8].

Accompanied with the network development are the various new techniques that improves transmission capacity significantly. To maximize the throughput over a fiber link, higher order modulation format adaptation [9], variable errorcorrecting code rate [10], and optimized launch power [11]

Research supported by the Swedish Research Council under Grant No. 2012-5280.

L. Yan and E. Agrell are with Chalmers University of Technology, Gothenburg, Sweden ( $\{$ lyaa,agrell $\} @$ chalmers.se). are used at the transceiver. Space-division multiplexing (SDM) has been proposed as a cost-effective alternative for singlemode fibers to increase the transmission data rate greatly [12]. The performance of these innovations can be evaluated on the link level by point-to-point transmission experiments, but their potential in the network context remains in part an unanswered question.

In this paper, we investigate the capacity and efficiency benefits of combining flexible-grid networks with different transmission techniques and new network features including modulation format and carrier frequency adaptation, adjustable launch power, variable coding rate, SDM, and regenerator placement. Some of these techniques aim at improving the network resource utilization, whereas others improve the fundamental transmission capacity of optical fibers. Resource allocation algorithms that ensure acceptable QoTs based on the GN model are reviewed. Numerical results are shown for the performance assessment.

\section{Physical LaYer ImPairment MOdel}

We consider transparent or translucent optical network composed of ideal colorless-directionless reconfigurable optical add-drop multiplexers (CD-ROADMs) nodes and bidirectional links with equal-length fiber spans. Erbium-doped fiber amplifiers (EDFA) are deployed at the end of each fiber span to compensate for the fiber loss. The linear impairments are compensated for at the receiver by digital signal processing. The PLIs are mainly attributed to the NLIs generated during signal propagation in fibers and the amplified spontaneous emission (ASE) noise from EDFAs.

According to the GN model [5], [6], given a set of demands $D$ in a flexible-grid network, the power spectral density (PSD) of the NLI per polarization for demand $i \in D$ can be expressed as [13]

$$
G_{i}^{\mathrm{SCI}}=\mu N_{i}^{\mathrm{span}} G_{i}^{3} \operatorname{arcsinh}\left(\rho \Delta f_{i}^{2}\right),
$$

and

$$
G_{i}^{\mathrm{XCI}}=\mu G_{i} \sum_{\substack{j \in D \\ j \neq i}} N_{i j}^{\mathrm{span}} G_{j}^{2} \ln \left(\frac{\left|f_{i}-f_{j}\right|+\Delta f_{j}}{\left|f_{i}-f_{j}\right|-\Delta f_{j}}\right),
$$

where $\mu=3 \gamma^{2} /\left(2 \pi \alpha\left|\beta_{2}\right|\right), \rho=\pi^{2}\left|\beta_{2}\right| / 2 \alpha, \gamma$ is the nonlinear parameter, $\alpha$ is the fiber power attenuation, $\beta_{2}$ is the group velocity dispersion parameter, and $f_{i}, \Delta f_{i}, G_{i}, G_{i}^{\mathrm{SCI}}$ and $G_{i}^{\mathrm{XCI}}$ are the center frequency, bandwidth, PSD, self-channel interference (SCI), and cross-channel interference (XCI) of demand $i \in D$, respectively. The ASE noise can be written as

$$
G_{i}^{\mathrm{ASE}}=N_{i}^{\mathrm{span}}\left(e^{\alpha L}-1\right) h \nu n_{\mathrm{sp}},
$$


where $L$ is the length per fiber span, $h$ is Planck's constant, $n_{\mathrm{sp}}$ is the spontaneous emission factor, and $\nu$ is the light frequency. If multicore fiber (MCF) is used, however, there will be additional intercore interference (ICI), which affects channels transmitted on overlapping spectrum and adjacent cores. The PSD of ICI is expressed as

$$
G_{i}^{\mathrm{ICI}}=\delta \sum_{j \in D_{i}} N_{i j}^{\mathrm{span}} G_{j}
$$

where $\delta$ is the ICI coefficient and $D_{i}$ is the set of demands that generate ICI to $i$.

Combining the ASE noise and NLIs, the requirement of the SNR for demand $i$ can be written as

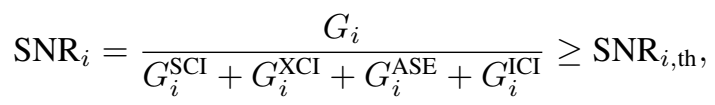

where $\mathrm{SNR}_{i}$ and $\mathrm{SNR}_{i \text {,th }}$ are the actual SNR and SNR threshold of demand $i$, respectively. If MCF is used, the $G_{i}^{\mathrm{ICI}}$ term will also appear in the denominator of (5). The SNR threshold depends on according the modulation format and error-correcting code used by the demand.

Note that in (1) and (2), the NLIs are nonlinear functions of the demand PSDs and spectrum assignments. The nonlinearity would be a computational burden to many resource allocation algorithms. This is because many problems, e.g., the assignment of route, spectrum, and regenerators, are modeled as mixed integer programming optimizations, which are solvable in realistic size networks only with linear constraints. Therefore, in order to leverage the precise NLI estimation of the GN model, it is necessary to simplify (1) and (2).

To approximate the GN model without much accuracy loss, linearization can be applied to (1) and (2). Mathematically, a function $h(\mathbf{x})$ can be fitted by a piecewise linear function [14] in the form of

$$
\hat{h}(\mathbf{x})=\max \left\{\mathbf{a}_{\mathbf{1}}^{\top} \mathbf{x}+b_{1}, \ldots, \mathbf{a}_{\mathbf{k}}^{\top} \mathbf{x}+b_{k}\right\},
$$

where $\mathbf{a}_{\mathbf{i}}^{\top}, b_{i}$ for $i=1, \ldots, k$ are the coefficients of a set of linear functions and $\mathrm{x}$ is a vector of resources to be optimized. In the resource allocation, $\mathbf{x}$ could be the assigned demand PSDs and frequencies. To obtain satisfactory QoTs, the linearized GN model should be an overestimation of the NLI PSDs, which implies $\hat{h}(\mathbf{x}) \geq h(\mathbf{x})$ for all meaningful $\mathbf{x}$. The number of linear functions $k$ controls the complexity and accuracy of the linearization. Thanks to the convexities of (1) and (2), good fitting qualities ${ }^{1}$ are achieved with $k=60$.

\section{CAPACITY ENHANCING TECHNIQUES IN FLEXIBLE-GRID NETWORKS}

In this section, we incorporate the GN model, new transmission techniques, and network features into flexible-grid networks. Their potential capacity and efficiency benefits are assessed by novel resource allocation algorithms and numerical results.

\footnotetext{
${ }^{1}$ The maximum relative fitting errors for SCI and XCI noises are $0.1 \%$ and $18.0 \%$, respectively, which are sufficient to yield good resource allocations in flexible-grid networks [14] since the contribution of XCI is usually comparatively small in the optimized resource allocations. A smaller fitting error can be achieved by setting $k$ to a higher value.
}

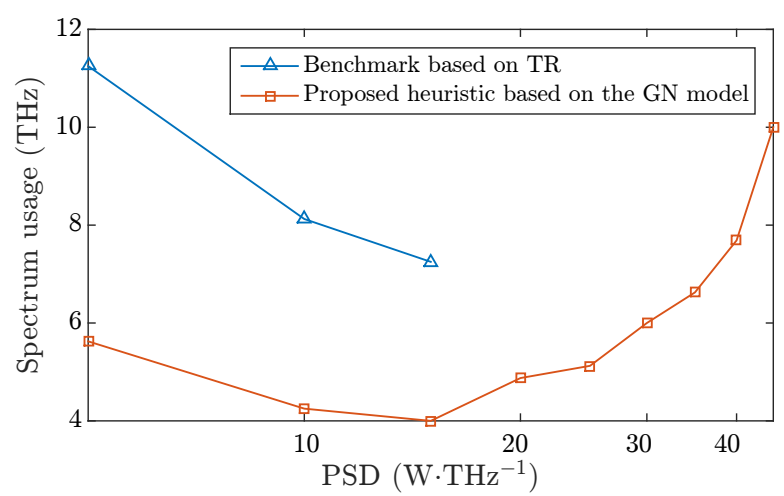

Figure 1: The spectrum usage of the proposed CL heuristic and the TR-based benchmark in the German network [8].

\section{A. Modulation Format and Carrier Frequency Adaptation}

Todays agile transponders can adapt the bit rate and spectrum usage by supporting several modulation formats and tunable center frequencies. With higher order quadrature amplitude modulation (QAM), an increased spectral efficiency of transmission is achieved at the price of higher SNR threshold and hence increased vulnerability to PLIs. Moreover, the coexistence of multiple modulation formats and channel bandwidths on fiber links would generate much higher spectrum fragmentation and NLIs, which makes it necessary to develop PLI-aware resource allocation algorithms.

To this end, we applied a connection list (CL) heuristic algorithm to solve the routing, modulation format, and spectrum allocation (RMSA) problem based on the GN model in flexible-grid networks [8], [15]. The CL heuristic allocates traffic demands one by one greedily. For each demand, the best routing, spectrum, and modulation format are chosen such that the QoTs of all the demands in the network are satisfactory. The order of processing demands is controlled by a simulated annealing heuristic [16]. A uniform PSD among all traffic demands is assumed. As is illustrated in Figure 1, by utilizing the GN model in the PLI estimation, the proposed CL heuristic improves the spectrum efficiency by $50 \%$ compared to the benchmark [17], which guarantees QoTs by limiting the route length to the worst case transmission reach (TR) for each modulation format. Moreover, the proposed heuristic can provide feasible solutions for all launch powers, whereas the benchmark works only at low power levels.

\section{B. Adjustable Launch Power}

A carefully selected uniform PSD has been shown to be important to efficient resource utilization in Figure 1. We push this even further by optimizing the PSD for each traffic demand [14]. The linearized GN model in the form of (6) is incorporated into a mixed integer linear programming (MILP) formulation to reduce the complexity. The proposed MILP is decomposed into three subproblems, which are solved sequentially to determine the routing, spectrum, and PSD per demand, respectively. The decomposition algorithm achieves fast running time and close-to-optimal performance by exploring the solution space with multiple start points. 


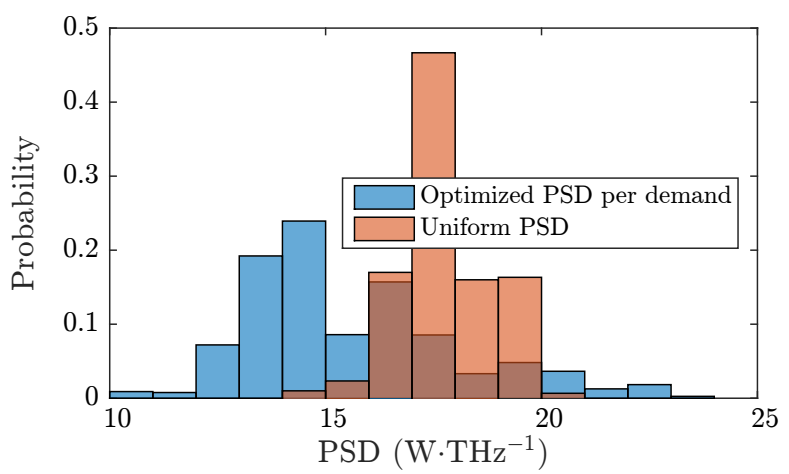

Figure 2: The distribution of allocated PSDs for the uniform and optimized PSD schemes in the German network.

The distribution of the individually optimized PSDs is compared with the uniform PSD scheme [8] in Figure 2 across multiple simulations, where each simulation will give a uniform PSD for the benchmark and different PSDs for each demands in the variable PSD method. The optimized PSD are spread in a large range, whereas the uniform PSD scheme has a much limited choice. In Figure 3, the relative gain of the optimized PSD scheme grows as the traffic load increases. This illustrates the effectiveness of the PSD optimization in heavily loaded networks, where the diverse NLIs generated by a large number of demands are mitigated significantly.

\section{Variable Coding Rate}

Forward error correction (FEC) enables the correction of errors introduced by noise or PLIs by adding redundancy to transmitted data in controlled fashion [18]. Transponders with variable modulation formats but fixed code rate can only provide discrete spectral efficiencies. In contrast, more degrees of freedom are offered by varying the FEC code rate as well.

In order to leverage the flexibility of variable coding rate, we propose a routing, code rate, modulation level, and spectrum assignment (RCMLSA) algorithm in the static network scenario [19]. In Figure 4, the spectrum utilizations of the RCMLSA and fixed coding rate resource allocation are shown, where RCMLSA achieves much better performance improvement over the whole range of transmit PSD.

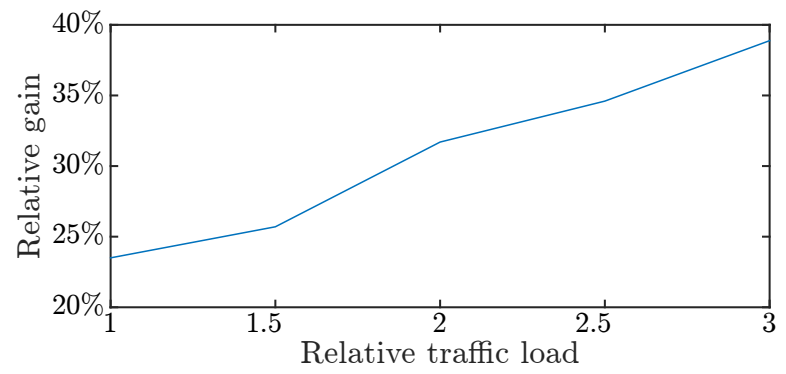

Figure 3: The gain of the optimized PSD relative to the uniform PSD scheme in the German network. The traffic load is measured relative to the single demand per node pair case.

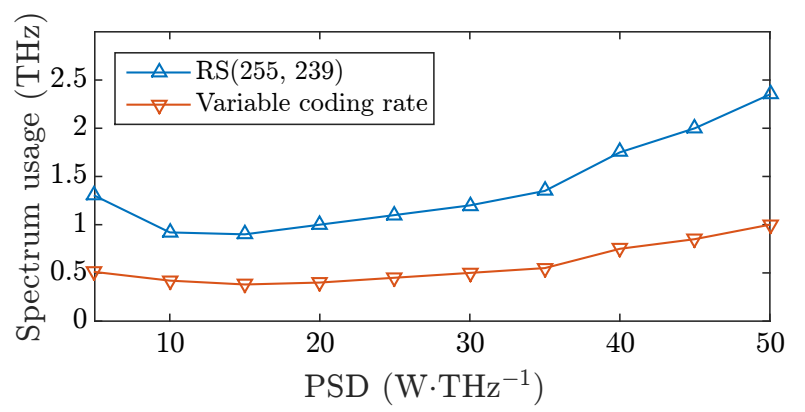

Figure 4: The spectrum usage comparison the fixed and variable coding rate in the German network. The Reed-Solomon (RS) code family is used.

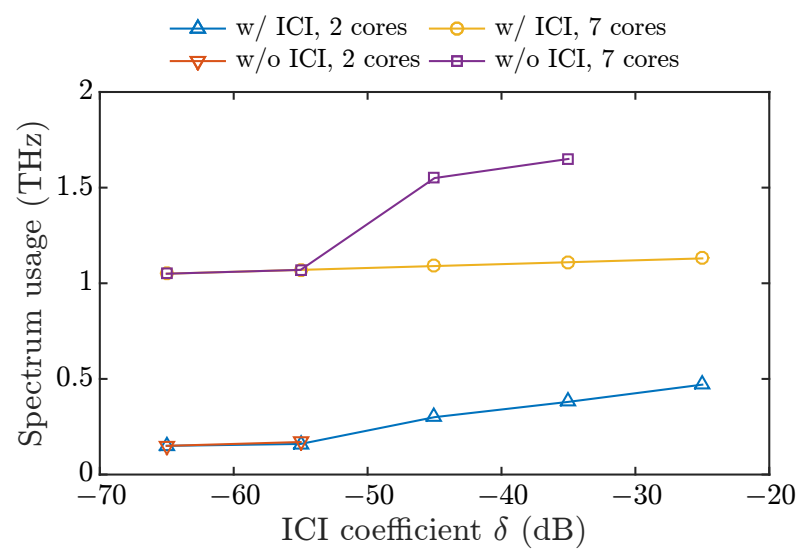

Figure 5: The impact of ICI on the spectrum usage in the German network.

\section{SDM}

Flexible-grid networks can provide higher throughput by using the existing resources more efficiently. The single-core fiber (SCF) underlying the physical layer, however, prevents the network capacity from fast growing. This limitation is circumvented by utilizing SDM to transmit parallel signals in the spatial dimensions of MCFs. To ensure acceptable QoTs, the ICI expressed in (4) should be considered in addition to the NLI and ASE noises.

To study the impact of ICI, we developed a spectrum and fiber core allocation scheme in MCF flexible-grid networks. We first sort the traffic demands in descending order of their data rates, and then assign core and spectrum to them one by one greedily with constraints that the QoTs of all the existing demands are always acceptable. We then simulate the network spectrum usage with variable ICI coefficient $\delta$ in 2core and 7-core MCF networks and compare the results with those neglecting ICI. As is shown in Figure 5, when $\delta \geq-55$ $\mathrm{dB} / 100 \mathrm{~km}$, the ICI must be considered in order to obtain feasible solutions for the 2-core MCF network, otherwise some demands will be blocked due to excessive ICI noise. A $30 \%$ spectrum reduction can be achieved in the 7-core MCF network when $\delta \geq-45 \mathrm{~dB} / 100 \mathrm{~km}$. 


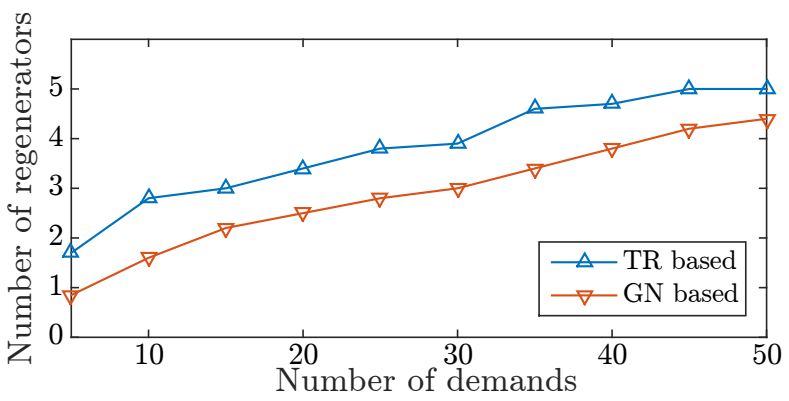

Figure 6: The average number of regenerators placed in the NSF-24 network [20] by the TR- and GN-based algorithms.

\section{E. Regenerator Placement}

In some submarine or large terrestrial networks, particularly if they involve high-order QAM or high baud rates, the length of the light path may be so long that the accumulated PLIs are too high to allow direct transmission. Regeneration is needed in intermediate nodes to restore the optical signals. The regenerators are, however, expensive since they require highspeed electronic equipment and maintenance by technicians. Therefore, accurate PLI estimation can help to optimize the regenerator placement such that proper QoTs are ensured in an economical fashion.

In our current work, we develop an iterative regenerator placement algorithm based on the decomposition of an MILP [20] and a linearized GN model. Our algorithm successfully reduces the complexity of MILP optimizations and can handle up to 50 concurrent traffic demands near-optimally. As illustrated in Figure 6, the linearized GN model estimates the PLIs accurately and achieves much conservative regeneration allocations compared with the TR-based method.

\section{CONCLUSION}

Significant capacity and efficiency improvements can be achieved by incorporating various new transmission techniques and network features into flexible-grid networks. The complicated and significant PLIs generated by coexisting heterogeneous optical signals on different wavelengths and/or cores in the network, however, should be precisely predicted. Our research shows that there is a great potential benefit in flexiblegrid networks, which could be harvested by carefully designed resource allocation algorithms based on accurate physical layer models.

\section{REFERENCES}

[1] M. Jinno, H. Takara, B. Kozicki, Y. Tsukishima, Y. Sone, and S. Matsuoka, "Spectrum-efficient and scalable elastic optical path network: Architecture, benefits, and enabling technologies," IEEE Communications Magazine, vol. 47, no. 11, pp. 66-73, 2009.

[2] A. Chralyvy, "Plenary paper: The coming capacity crunch," in Proc. European Conference on Optical Communication (ECOC), Vienna, Austria, Sept. 2009.

[3] D. J. Richardson, "Filling the light pipe," Science, vol. 330, no. 6002, pp. 327-328, 2010.
[4] O. Gerstel, M. Jinno, A. Lord, and S. Yoo, "Elastic optical networking: A new dawn for the optical layer?" IEEE Communications Magazine, vol. 50, no. 2, pp. 12-20, 2012

[5] P. Poggiolini, G. Bosco, A. Carena, V. Curri, Y. Jiang, and F. Forghieri, "The GN-model of fiber non-linear propagation and its applications," IEEE Journal of Lightwave Technology, vol. 32, no. 4, pp. 694-721, 2014.

[6] P. Johannisson and E. Agrell, "Modeling of nonlinear signal distortion in fiber-optic networks," IEEE Journal of Lightwave Technology, vol. 32, no. 23, pp. 3942-3950, 2014.

[7] L. Yan, E. Agrell, H. Wymeersch, P. Johannisson, R. Di Taranto, and M. Brandt-Pearce, "Link-level resource allocation for flexiblegrid nonlinear fiber-optic communication systems," IEEE Photonics Technology Letters, vol. 27, no. 12, pp. 1250-1253, June 2015.

[8] J. Zhao, H. Wymeersch, and E. Agrell, "Nonlinear impairment-aware static resource allocation in elastic optical networks," IEEE Journal of Lightwave Technology, vol. 33, no. 22, pp. 4554-4564, 2015.

[9] C. Laperle and K. Roberts, "Flexible transceivers," in Proc. European Conference on Optical Communication (ECOC), Amsterdam, Netherland, Sept. 2012, p. We.3.A.3.

[10] L. Beygi, E. Agrell, J. M. Kahn, and M. Karlsson, "Rate-adaptive coded modulation for fiber-optic communications," IEEE Journal of Lightwave Technology, vol. 32, no. 2, pp. 333-343, 2014.

[11] V. Curri, A. Carena, A. Arduino, G. Bosco, P. Poggiolini, A. Nespola, and F. Forghieri, "Design strategies and merit of system parameters for uniform uncompensated links supporting Nyquist-WDM transmission," IEEE Journal of Lightwave Technology, vol. 33, no. 18, pp. 3921-3932, 2015.

[12] B. Puttnam, R. Luis, J. Sakaguchi, W. Klaus, J.-M. D. Mendinueta, Y. Awaji, N. Wada, and E. Agrell, "Pb/s, homogeneous, single-mode, multi-core fiber systems," in Proc. European Conference on Optical Communication (ECOC), Düsseldorf, Germany, Sept. 2016, p. Tu.1.D.2.

[13] L. Yan, E. Agrell, H. Wymeersch, and M. Brandt-Pearce, "Resource allocation for flexible-grid optical networks with nonlinear channel model," Journal of Optical Communications and Networking, vol. 7, no. 11, pp. B101-B108, 2015.

[14] L. Yan, E. Agrell, M. N. Dharmaweera, and H. Wymeersch, "Joint assignment of power, routing, and spectrum in static flexible-grid networks," IEEE Journal of Lightwave Technology, to be published.

[15] J. Zhao, H. Wymeersch, and E. Agrell, "Nonlinear impairment aware resource allocation in elastic optical networks," in Proc. Optical Fiber Communication Conference (OFC), Los Angeles, CA, Mar. 2015, pp. M2I-1.

[16] M. Aibin and K. Walkowiak, "Simulated annealing algorithm for optimization of elastic optical networks with unicast and anycast traffic," in Proc. International Conference on Transparent Optical Networks (ICTON), Graz, Austria, July 2014, p. Th.B3.6.

[17] K. Christodoulopoulos, I. Tomkos, and E. Varvarigos, "Elastic bandwidth allocation in flexible OFDM-based optical networks," IEEE Journal of Lightwave Technology, vol. 29, no. 9, pp. 1354-1366, 2011.

[18] E. Agrell, M. Karlsson, A. Chraplyvy, D. J. Richardson, P. M. Krummrich, P. Winzer, K. Roberts, J. K. Fischer, S. J. Savory, B. J. Eggleton et al., "Roadmap of optical communications," Journal of Optics, vol. 18, no. 6, p. 063002, 2016.

[19] J. Zhao, L. Yan, H. Wymeersch, and E. Agrell, "Code rate optimization in elastic optical networks," in Proc. European Conference on Optical Communication (ECOC), Valencia, Spain, Sept. 2015, p. We.3.5.1.

[20] X. Wang, M. Brandt-Pearce, and S. Subramaniam, "Impact of wavelength and modulation conversion on transluscent elastic optical networks using MILP," Journal of Optical Communications and Networking, vol. 7, no. 7, pp. 644-655, 2015. 